\title{
Tap-and-2-Split Switch Design Based on Integrated Optics for Light-Tree Routing in WDM Networks
}

\author{
Gonzalo M. Fernández, Student Member, IEEE, Carmen Vázquez, Senior Member, IEEE, \\ Pedro Contreras Lallana, Student Member, IEEE, and David Larrabeiti, Member, IEEE
}

\begin{abstract}
This paper presents a novel cost-effective multicastcapable optical cross connect (MC-OXC) node architecture that features both tap-and-continue and tap-and-binary-split functionality. This architecture provides an interesting balance between simplicity, power efficiency and overall wavelength consumption with respect to models based on $\mathrm{TaC}$ (Tap and Continue) or $\mathrm{SaD}$ (Split-and-Delivery). The main component of this node is a novel Tap-and-2-Split Switch (Ta2S). In this paper, we propose and analyse an implementation of this switch based on integrated optics (namely, MMI taps and MZI switches), and we characterize and compare it with other alternatives implemented with the same technology. The study shows that, thanks to the presented Ta2S design, the 2-Split Tap Continue (2STC) node scales better in terms of number of components than the other alternatives. Moreover, it is more power efficient than the $\mathrm{SaD}$ design and requires less wavelengths than TaC thanks to the binary split capability. On the other hand, simulation results reveal that the 2 -split condition does not add a significant additional wavelength consumption in usual network topologies with respect to SaD.
\end{abstract}

Index Terms-All-optical multicast routing, light-trees, multicast node architectures, splitters, wavelength routed multicast.

\section{INTRODUCTION}

$\mathbf{M}$ ANY data applications such as high-definition television (HDTV), video-on-demand (VOD), virtual private LAN service (VPLS), GRID computing, optical storage area networks (O-SAN), etc., can take advantage of multicast communication [1]. The multipoint service can be cost-effectively exploited at the optical layer when very high data rates are required. This is the case, for instance, of scientific applications requiring bulk data distribution among networked supercomputers, but also the case of large aggregates of multicast/broadcast/unknown virtual LAN traffic whose broadcast functionality must be emulated by the VPN service provider. In order to realize optical multicast, the lightpath (all-optical point-to-point path) concept was generalized in [2] to that of light-tree, which consists of an optical transparent point-to-multipoint channel

Manuscript received May 28, 2008; revised November 13, 2008. Current version published July 01, 2009. This work was supported in part by the BONEproject ("Building the Future Optical Network in Europe"), a Network of Excellence funded by the European Commission through the 7th ICT-Framework Program and in part by the Spanish Ministry of Education and Science Grants TEC2006-13273-C03 03 and TSI2005-07384-C03-02.

G. M. Fernández and D. Larrabeiti are with Department of Telematics Engineering, Universidad Carlos III de Madrid, 28911 Madrid, Spain (e-mail: gmfernand@hotmail.com; dlarra@it.uc3m.es).

C. Vázquez and P. C. Lallana are with Department of Electronics Technology, Universidad Carlos III de Madrid, 28911 Madrid, (e-mail: cvazquez@ing.uc3.es; pcontrer@ing.uc3m.es).

Digital Object Identifier 10.1109/JLT.2008.2012174 originated at a source node that has more than one destination node. A light-tree spans several nodes and fiber links, and it is independent to bit-rate, protocol and format [3], and eliminates the need of per-hop packet forwarding. An excellent survey of algorithms for light-tree construction (a.k.a. light-tree routing) over WDM networks can be found in [1].

The key element of light-tree routing is the optical multipoint capability of optical switches. Several multicast-capable node architectures have been proposed. One is the $\mathrm{SaD}$ (Split and Delivery) architecture [4], [5], which is based on fixed or configurable power splitters [6] that require optical amplication. From that initial architecture, several other structures were proposed, either focused on improving power efficiency or on fabrication cost. One of these improvements came through the split-sharing concept e.g., the MOSAD architecture. This concept prevents splitting for non-multicast cross-connections but it has the same power penalty as $\mathrm{SaD}$ for multi-point connections going through the shared splitter and, the saving in splitters comes at the price of internal blocking probability. Another approach is the $\mathrm{TaC}$ node (Tap-and-continue node) [7], which improves power efficiency by tapping a small amount of the signal power to the local node when it is a leaf in the multicast tree. Tapping reduces the need for optical amplification at the cost of suboptimal light-tree construction, which, depending on the network topology, can lead to considerable over-occupation of wavelengths.

Since both $\mathrm{SaD}$ and $\mathrm{TaC}$ seem to be extremely opposite alternatives, in [15] we firstly drafted a novel node architecture that tries to combine the advantages of both tapping and splitting. In this paper we continue this work and focus on the implementation of the switch that constitutes the key element of the architecture, by means of state-of-the-art integrated optics. This node-named 2-STC-is designed to both perform tapand-continue and tap-and-2-split. We analyse the power efficiency of this element and compare it numerically with $\mathrm{SaD}$ and $\mathrm{TaC}$, using as reference their respective implementation on integrated optics.

The study reveals: a) that binary splitting combined with tapping provides a fair improvement in wavelength utilization over $\mathrm{TaC}$ in usual topologies, b) that the cost of the node in number of components is below $\mathrm{TaC}$ [7], c) that given a power budget it is possible to reach a number of leaves without amplification close to $\mathrm{TaC}$, and d) that the excess attenuation (i.e., insertion loss excluding the splitting ratio) is far below the other architectures. The key to some of these properties is a novel network of $2 \times 1$ switches inside the Ta2S switch. 


\section{Data Plane and Control Plane of Optical Multicast}

\section{A. Data Plane: Multicast OXC Node Architectures}

SaD-Based MC-OXC Node Architectures: The split-and-delivery switch ( $\mathrm{SaD}$ switch) was first proposed in [4] as the main component of the SaD-based multicast capable node. This architecture was further modified in [5] in order to reduce its cost and to improve power efficiency. A completely optical multicast capable OXC node (MC-OXC) would be mainly composed of passive optical light splitters to enable multicasting, which divide the input power into several outputs without any knowledge about the optical features of the input signal and without changing any property of it except the power. A split operation contributes to power loss; for an ideal device the power of each output is the $(1 / n)$-th part of the input signal.

An improvement to the $S a D$-switch was later proposed in [6] that employed configurable splitters instead of passive splitters. Fig. 1(a) represents a $P \times P \mathrm{SaD}$ switch. These devices can be instructed to split the incoming signal into $m$ outputs $(m \in$ $\{1, \ldots, P\})$, where $m=1$ corresponds to no splitting and $m=$ $P$ to a broadcast operation. After splitting, each of the $m$ split resulting signals may be switched to the correspondent output by using a $P^{2}$ photonic switch matrix made up of $2 \times 1$ optical switching elements. As shown in Fig. 1(a), $P^{2}$ optical amplifiers are required. They are located after the splitter so that each split signal is optically amplified to compensate the power loss due to splitting. Some known drawbacks of optical amplifiers are their high cost, complex fabrication and amplification of noise levels.

Several other SaD-based node architectures were proposed in [4]. Some of these proposals include built-in wavelength conversion capability i.e., nodes can set up point-to-multipoint connections to different output wavelengths. This capability improves overall blocking performance; however, for the sake of simplicity of comparison, in this work we shall refer only to singlewavelength splitting nodes. Equivalent designs could easily be obtained by placing wavelength converters at the outputs.

The basic and most well-known architecture is that of Fig. 1(b), which is composed of a set of $W P \times P \mathrm{SaD}$ switches (with $W=$ number of wavelengths). Each input fiber is demultiplexed to extract the individual wavelengths, which are then directed to their corresponding $\mathrm{SaD}$ switches, where splitting and space switching operations take place. Finally, $P$ multiplexers combine the $W$ signals onto their corresponding outgoing fibers. Other architectures improve power efficiency for cross-connections that do not require splitting [5] by applying the splitting-sharing concept. In this approach, all requests share a single power splitter that is attached to one of the switch ports. Therefore, only one multicast request can be attended at a time. It reduces costs and complexity, but it has poorer performance in terms of blocking probability for multi-point connections. Furthermore, multicast connections suffer the same process as in $\mathrm{SaD}$, and hence require amplification. On the other hand, unicast connections do not have to traverse the splitter, which is a certain advantage.

Tap-and-Continue MC-OXC Node Architecture: With the aim of reducing the cost and improve the power efficiency of MC-OXCs a new architecture called Tap-and-Continue

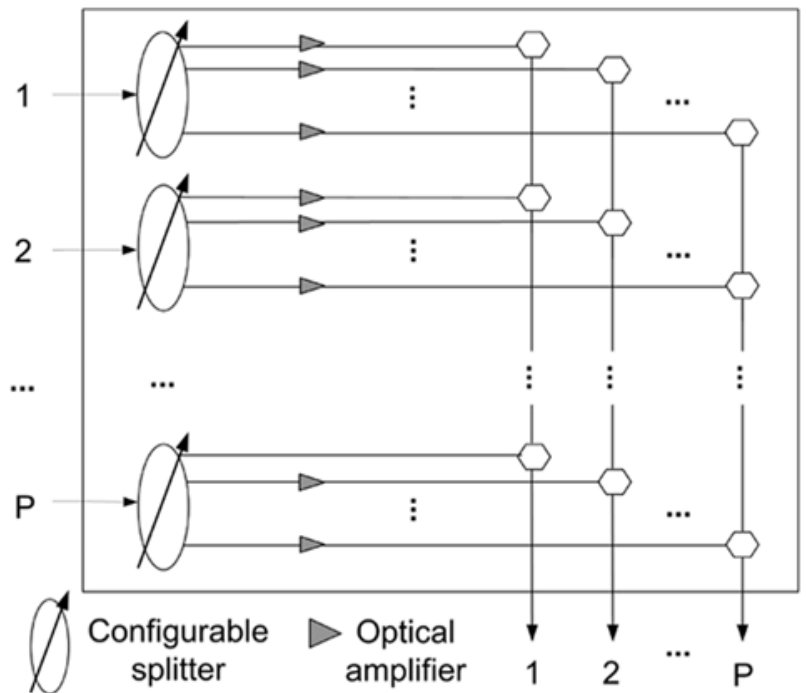

(a)

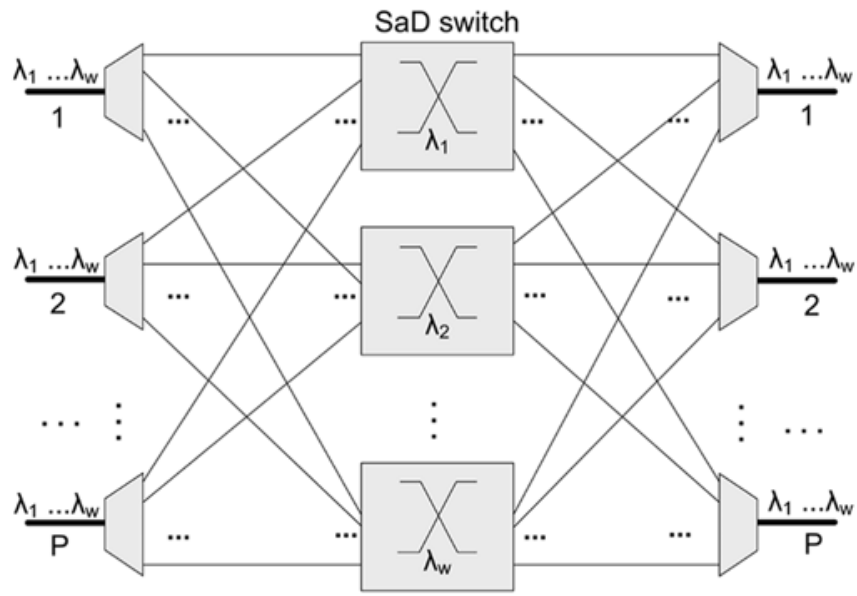

(b)

Fig. 1. SaD switch and SaD-based node architecture [4], [5]. (a) SaD switch with $P$ inputs and $P$ outputs. (b) MC-OXC architecture based on $\mathrm{SaD}$ switches.

(TaC) was proposed in [7]. The purpose of this architecture is doing without splitters to reduce the need for amplifiers in the network. In this approach, when the node is an end-point of the multi-point connection, only a very small fraction $(0.5 \%-10 \%)$ of the incoming power is tapped locally and the rest is switched to an output port. This fraction is assumed to be enough for the local detector and most of the power is preserved for transmission to other nodes. This architecture contains, per each wavelength, a $(P+1) P$ wavelength routing switching (WRS) module and one shared tap-and-continue module (TCM), which taps to the local node. Fig. 2(a) depicts the node and Fig. 2(b) depicts the TCM. As can be seen in the figure, the selected design is actually shared tapping.

The main disadvantage of this architecture comes from the lack of splitting functionality. In $\mathrm{TaC}$ the light-tree becomes a linear graph passing through all the nodes in the multi-point connection. This makes it appropriate for ring-like topologies but rather suboptimal in meshed topologies in terms of used wavelengths and average delay. Multicast connections can be realized by using only these TaC OXC nodes, and multicast routing 


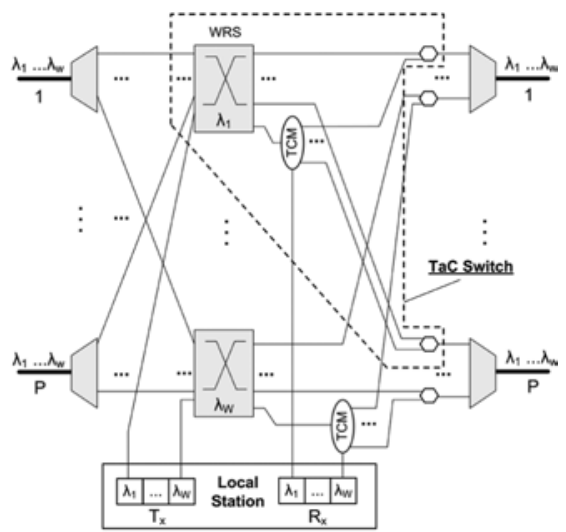

(a)

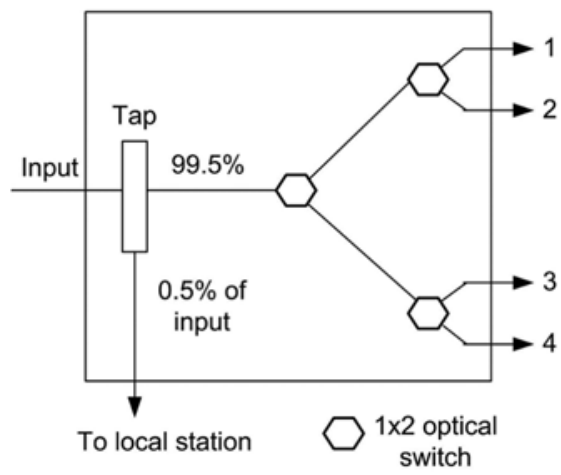

(b)

Fig. 2. Tap-and-continue node and TaC module (TCM) [5], [7]. (a) A $P \times P$ TaC-OXC node architecture. (b) A $1 \times 4$ TCM.

algorithms for networks composed of these nodes have been proposed in [5], [8]. A mixed utilization of splitters $(\mathrm{SaD})$ and tapping $(\mathrm{TaC})$ in different parts of the network has been devised as the only intermediate approach to overcome the cons of $\mathrm{TaC}$.

\section{B. Control Plane: MC-RWA and Power Loss Constraints}

Regarding the control plane, the major issue to solve is the multicast routing and wavelength assignment (MC-RWA) problem. The problem of finding the optimal light-tree is NP-complete and is formalized as the Steiner tree problem [16]. There is much research work for the MC-RWA problem, and proposed solutions deal with MC-RWA for a single multicast request, multiple static multicast requests, and multiple dynamic multicast requests. A wide state of the art can be found in [1]. Most of the approaches are focused on reducing used wavelengths, max-min-avg distance from source node to destinations, etc. In this paper we concentrate on power.

The splitting of an optical signal implies a considerable power loss that leads to a bound to the times a signal can be split. Furthermore since optical amplifiers also amplify noise levels, it can be assumed that there is also an upper bound on optical amplification [5], [9]. In other words, reducing the amount of amplifiers in the network does not simply obey to monetary cost. There is also the fiber link attenuation. The problem of building light-trees under such optical power constraints [9], [10] is not the focus of this paper. A balanced tree is found to be the optimum setting in $\mathrm{SaD}$. In the case of $\mathrm{TaC}$, the problem is reduced

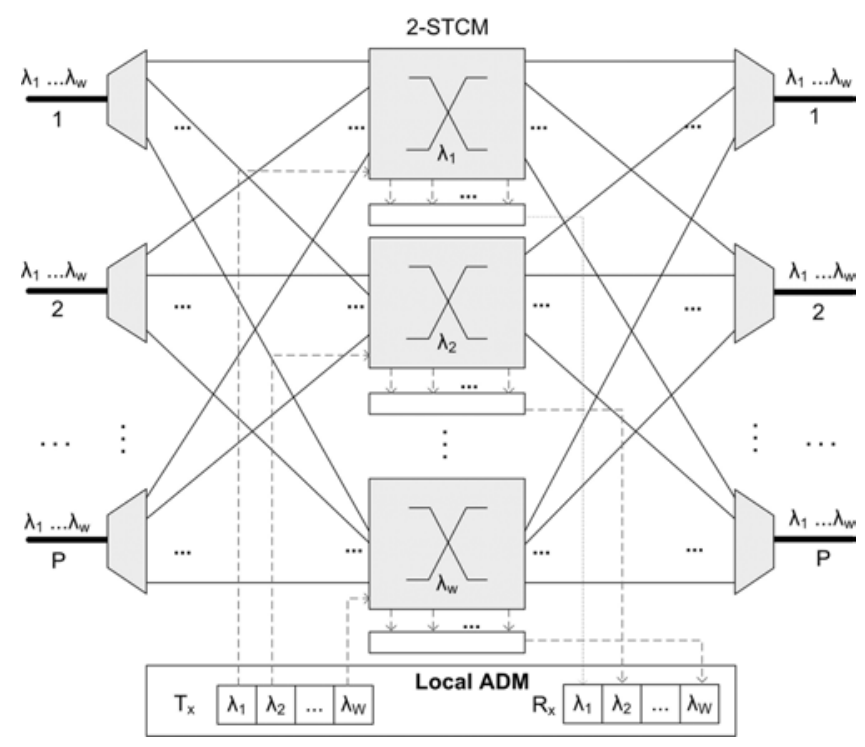

Fig. 3. General architecture of a $\mathrm{P} \times \mathrm{P} 2$-STC MC-OXC node with novel 2-STCM.

to the open traveling salesman problem. We shall use known heuristics to search for both optimal directed graphs for $\mathrm{SaD}$ and $\mathrm{TaC}$ in the simulations.

In this paper we propose to limit the split fan-out to two branches, and combine it with optional tapping by means of integrated optics. This represents another variant of the MC-RWA problem. In this case, the target is building binary trees as balanced as possible in order to maximize the number of nodes that receive enough power for effective tapping. We claim that this approach shows an ideal trade-off between simplicity of implementation (optimal by tapping in $\mathrm{TaC}$ ) and transmission costs (optimal by splitting in $\mathrm{SaD}$ ).

\section{The Novel 2-SPlit-TAP-Continue NODE ARCHITECTURE}

\section{A. General Node Architecture}

We aim to combine the advantages of $\mathrm{TaC}$ and $\mathrm{SaD}$ in a costeffective design based on integrated optics. Fig. 3 depicts the general architecture for the 2-split-tap-continue node (2-STC node) firstly sketched by the authors in [15]. The node has the following capabilities.

- Tap-and-continue ( $\mathrm{TaC})$ : Besides switching, it taps a small fraction of the input power to the local node. Unlike $\mathrm{TaC}$ nodes [7] described above, tap is always performed. In $\mathrm{TaC}$ nodes, the tap is optional, but it increases the number of switches needed.

- Tap-and-2-split (Ta2S): The node should tap and perform 2-split for multiple requests in a strictly non-blocking manner. As described, tap is always performed.

The general architecture is very similar to a SaD-based node [4], [5], but instead of $\mathrm{SaD}$ switches, a novel module 2-Split-Tap-Continue Module (2-STCM) is used, which will be explained later. It needs $P$ multiplexers to extract individual wavelengths from each input fiber and $P$ demultiplexers to combine individual wavelengths onto output fibers. A matrix for receiving taps is used $\left(R_{x}\right)$ at the Add-Drop Multiplexer 


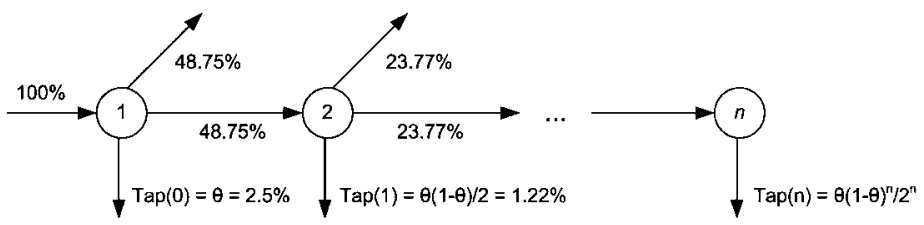

(a)

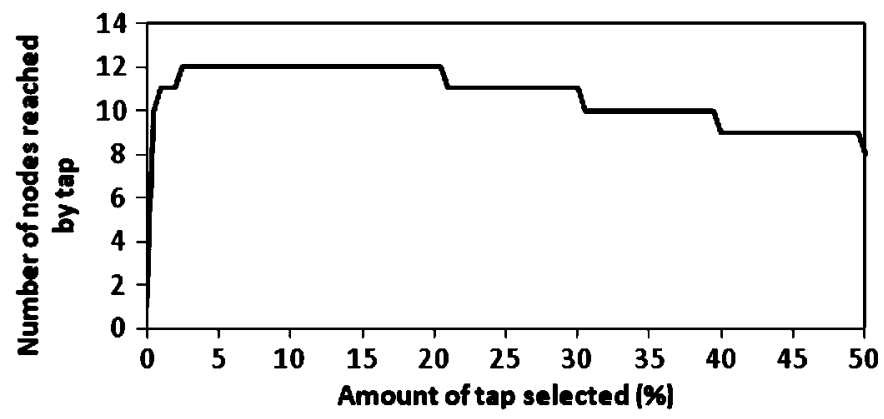

(b)

Fig. 4. Theoretical outreach of tapped power on a branch with tap-2-splitting. (a) Ratio of input power tapped in a branch made up of $n$ nodes performing tap-and-2-splitting, with a fixed tap of $2.5 \%$. (b) Number of nodes of the branch that receive the tapped signal with less than $51 \mathrm{~dB}$ of attenuation versus fixed selected tap rate $\%$

(ADM) local station, and it could be necessary to have a selector in every 2-STCM because the tap operation is made for every input port.

\section{B. The 2-Split-Tap-Continue Module (2-STCM)}

Tap-and-2Split Switch (Ta2S Switch): The 2-split operation is the key of our proposal for two reasons: (a) realizing 2-split saves power efficiency, since it divides the incoming power only into two outputs. We will show later that, in generic core networks, the 2-split condition is sufficient and achieves a good trade-off between power efficiency and the number of resources used; and (b) a 2-split operation is much easier to perform than an $n$-split one $(n>2)$, in terms of used components, complexity and fabrication tolerances.

Therefore, we need a device with a single input signal and two output signals, able to switch to any of the outputs $(0: 100$ or 100:0), or to share the optical power to both outputs (50:50); and has also to be able to tap a fixed value of the incoming signal. This novel device is named Tap-and-2-Split switch (Ta2S switch) that can be made with existing photonic devices.

The fixed tap value has been determined as follows. Let us consider a power budget permitting $51 \mathrm{~dB}$ of attenuation. That power is at $100 \%$ at the root of the light-tree shown in Fig. 4(a). The signal has to traverse $n$ nodes performing tap-and-2-splitting until it can not be detected by the $(n+1)$ th node. Fiber attenuation on the links, power amplification, power loss generated by nodes are not considered except those caused by the binary splitting $(3 \mathrm{~dB})$. Different values of fixed tap (at every node) have been tried out in order to find the most adequate one. Results are shown in Fig. 4(b), and it can be seen that the best performance is achieved when the fixed amount of tap at every node is set between $2.5 \%-20.5 \%$ (tap can be detected until the 12 th node). For our calculations, a fixed amount of $6 \%$ tap will be considered.

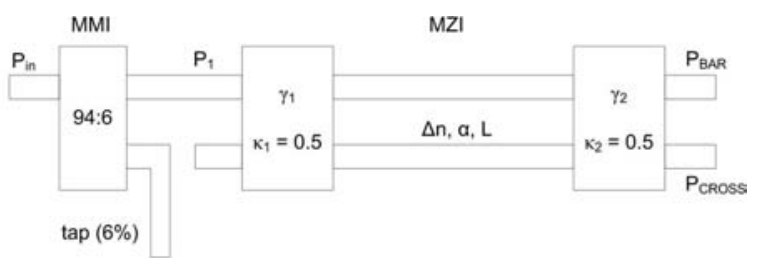

(a)

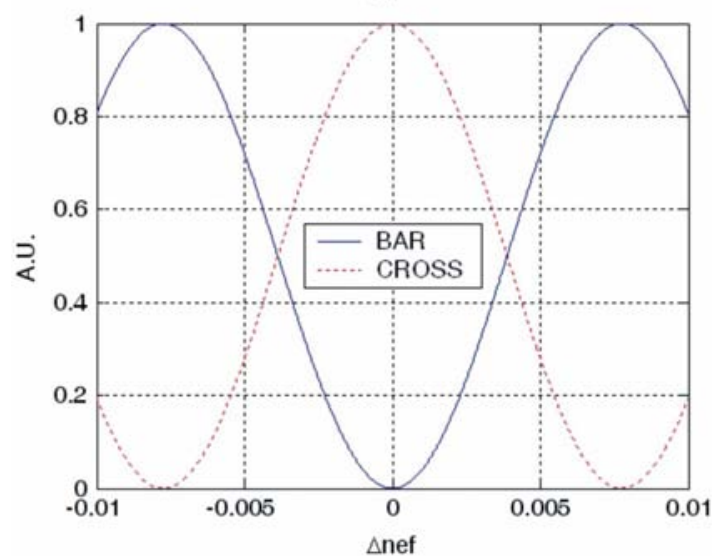

(b)

Fig. 5. Schematic of a Ta2S switch and output power response when $\Delta n$ modified. (a) Schematic of a Ta2S switch composed of a fixed splitter and a MZI. (b) Bar and Cross Outputs of MZI versus $\Delta n$.

An implementation of a Ta2S using a MZI configuration and a fixed tap is shown in Fig. 5.

The first stage of the Ta2S switch block diagram is a 94:6 splitter to perform the tap operation. The proposed optical switch is based on a MZI, shown on the right in Fig. 5(a). It is formed by two $3 \mathrm{~dB}$ couplers, with coupling ratios $\kappa_{1}=\kappa_{2}=0.5$ and excess losses $\gamma_{1}$ and $\gamma_{2}$, joint by two waveguides of length $L$ and attenuation $\alpha$. The switch output is selected by modifying the refractive index difference between both waveguides, $\Delta n$.

Thus, when $\Delta n=0$, at initial setup, all the optical input power is guided to the $P_{\text {CROSS }}$ output port. When the refractive index difference is induced, either by applying current or by thermo-optic effect, the optical power at both output ports changes. Output power variations, $P_{\mathrm{BAR}}$ and $P_{\mathrm{CROSS}}$, with the refractive index difference changes between the waveguides are shown in Fig. 5(b). Outputs are symmetrical, a maximum in cross output coincides with a minimum in bar output, and the optical power at each port can be adjusted modifying $\Delta n$.

A compact solution can be realized using Multi Mode Interference (MMI) splitter for the tap [11] and $3 \mathrm{~dB}$ MMI couplers [12] in the MZI. The MMI operation is based on the property of self image of the light propagation in planar waweguides. A MMI coupler consists of two inputs and two outputs attached to a wider section waveguide whose length determines the coupling ratio. The proposed $3 \mathrm{~dB}$ MMI coupler has input and output waveguides placed at a third part of the MMI width in order to obtain a shorter device, and its length is given by

$$
L_{\mathrm{MMI}}(3 \mathrm{~dB})=\frac{1}{2} L_{\pi}=\frac{1}{2} \frac{4 \cdot W_{e}^{2} \cdot n}{3 \cdot \lambda_{0}}
$$




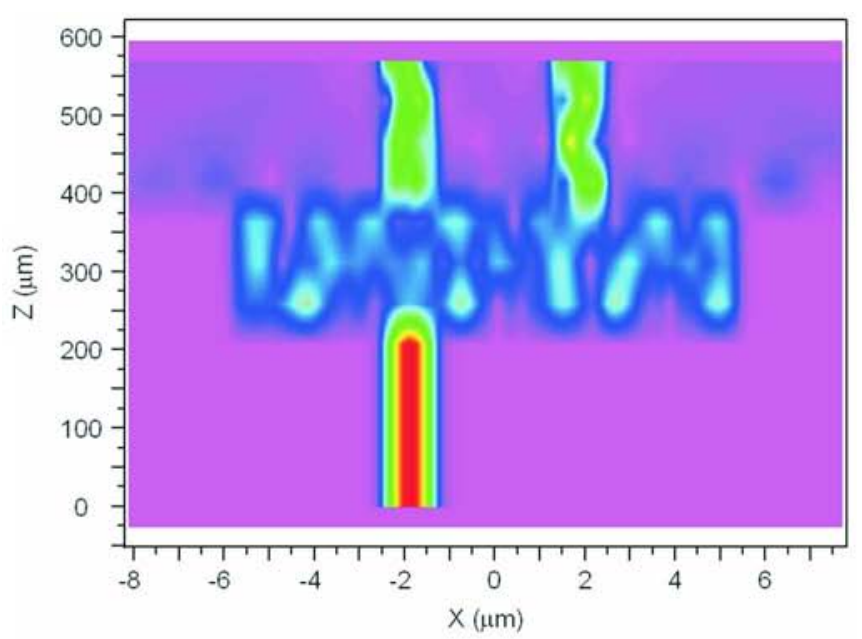

(a)

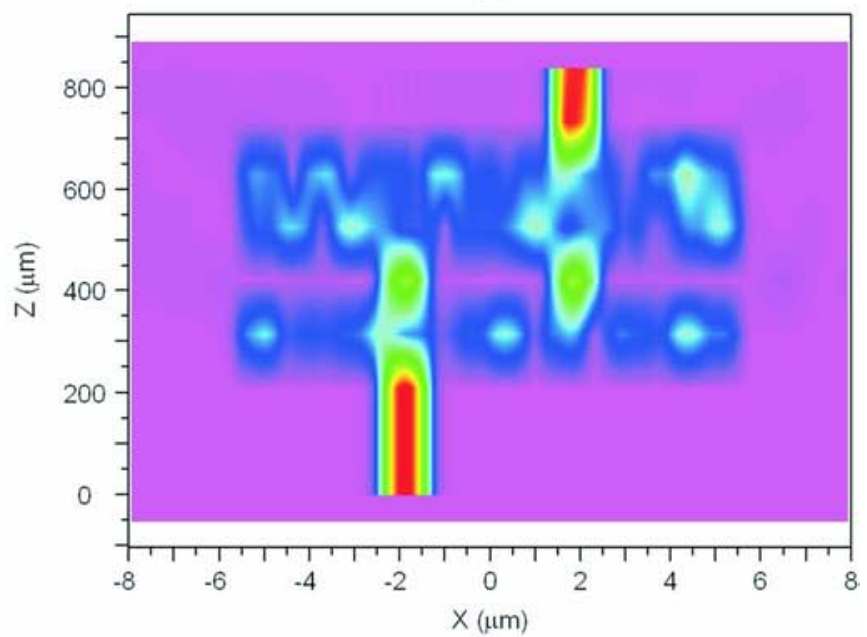

(b)

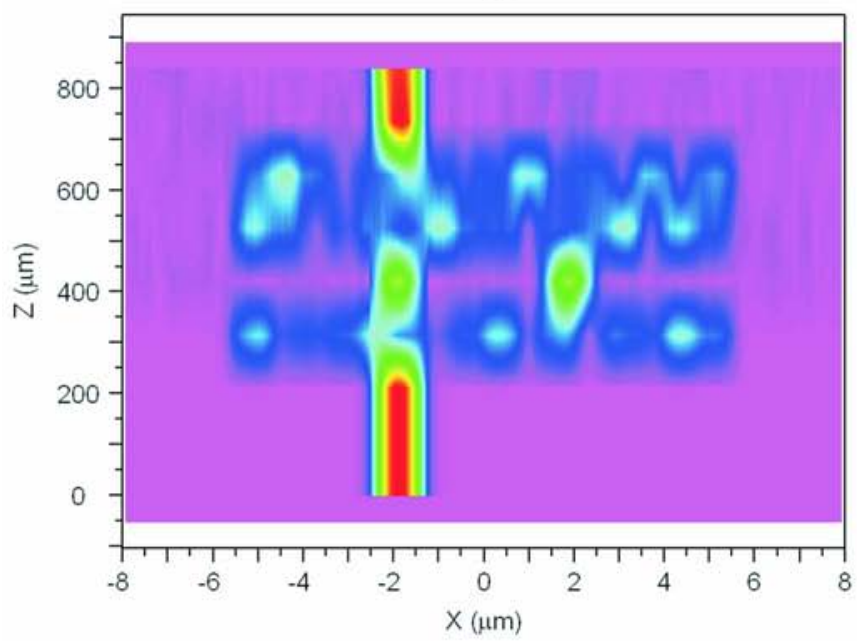

(c)

Fig. 6. Distribution of the optical power for $3 \mathrm{~dB}$ MMI and MZI switch. (a) Distribution of the optical power in a MMI $3 \mathrm{~dB}$ coupler. (b) Distribution of the optical power in MZI at initial status. (c) Distribution of the optical power when the MZI is switched.

where $L_{\pi}$ is the beat length, $W_{e}$ is the MMI width, $\lambda_{0}$ is the vacuum wavelength and $n$ is the effective refractive index of the waveguide [11], [12].

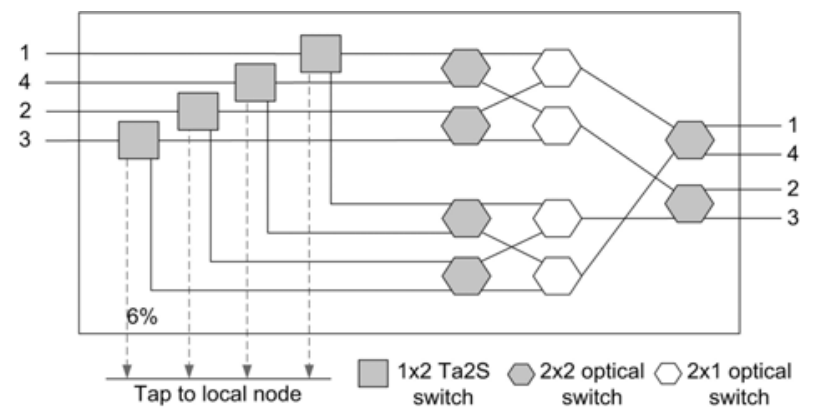

(a)

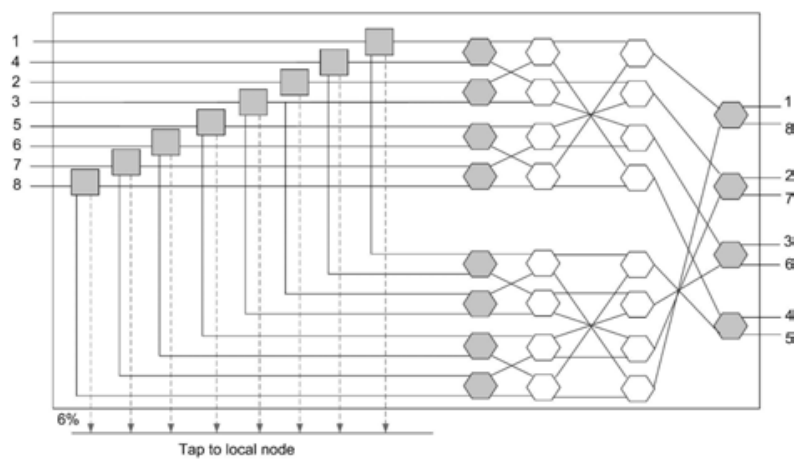

(b)

Fig. 7. Block diagram of the 2-STCM. Input and output ports are arranged for easy representation. (a) $4 \times 42$-STCM. (b) $8 \times 82$-STCM.

Simulations using the Beam Propagation Method (BPM) at $\lambda_{0}=1550 \mathrm{~nm}$ were carried out in order to show the operation principle. The effective refractive index of the ridge waveguides was 3 . The waveguides were $1.3 \mu \mathrm{m}$ wide, and the $3 \mathrm{~dB}$ MMI couplers are $11.3 \mu \mathrm{m}$ wide and $170 \mu \mathrm{m}$ long. A detail of the optical power distribution in the $3 \mathrm{~dB}$ MMI is shown in Fig. 6(a). Two simulations of the MZI with $100 \mu \mathrm{m}$ long waveguides between the two couplers are shown in Fig. 6(b) and (c). The first one shows the initial status, while the second one correspond to $\Delta \mathrm{n}=-0.00775$ simulation. The output for both cases has changed.

The Ta2S can also be realized using a tunable splitter [13], a $2 \times 2$ MMI with symmetric splitting ratios.

Block Diagram and Components: Fig. 7 shows $4 \times 4$ and $8 \times 82$-STC module block diagrams. The $P$ input signals go through $P$ Ta2S switches, which are instructed to perform the required operation. The resulting signals traverse $\log _{2} P+1$ phases of switches instructed adequately to guide them to the desired ports.

Note that the optical switching stage here designed is not similar to a typical $N \times M$ crossbar switch matrix (where $N=$ $2 P, M=P$ ). In such a matrix the best case of a switch operation is given when the optical signal traverses only one switch, the worst case when it has to traverse $N+M-1$ switches, and an average case, $(N+M) / 2$ switches. The interconnection network presented here is designed to use the least possible number of switches. Thus, a signal always traverses a fixed amount of $\log _{2} P+1$ switches (number of phases).

For a $P \times P / 2$ matrix, if $P$ is a power of 2 , each of the first $\log _{2} P$ phases has $P$ switches and the last one $P / 2$. If that is not the case, a $P^{\prime} \times P^{\prime} / 2$ switch matrix would be given (with 


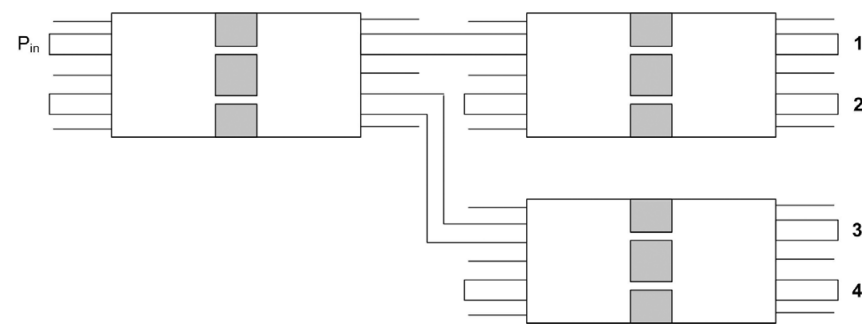

Fig. 8 . A $1 \times 4$ configurable splitter using $2 \times 2$ MMIs with symmetric splitting ratios.

TABLE I

POWER LOSS (DB) IN THE CONFIGURABLE SPLITTER OF FIG. 8

\begin{tabular}{lccc}
\hline Selected output ports & $\begin{array}{c}\text { Loss in } 1^{\text {st }} \\
\text { phase }\end{array}$ & $\begin{array}{c}\text { Loss in } 2^{\text {nd }} \\
\text { phase }\end{array}$ & $\begin{array}{c}\text { Total } \\
\text { loss }\end{array}$ \\
\hline $1,2,3$ and 4 & 0.1 & 0.1 & 0.2 \\
$1,2,3$ or 4 & 1.55 & 1.55 & 3.10 \\
1 and 2 , or 3 and 4 & 1.55 & 0.1 & 1.65 \\
1 and 3 , or 2 and 4 & 0.1 & 3.10 & 3.20 \\
$1,2,3$, or $2,3,4$ & 0.1 & 1.55 & 1.65 \\
\hline
\end{tabular}

$P^{\prime}=2^{\left\lceil\log _{2} P\right\rceil}$, and then it should be pruned in order to remove the unnecessary switches to get exactly what is needed for $P$ ports. In order to get more compact designs in the future, those switches can also be implemented on the same chip using ring resonators [14].

\section{Evaluation of Number of COMPONENTS AND POWER LOSS}

Let us now analyze the different factors of attenuation in the three basic node architectures under study, based on $\mathrm{SaD}, \mathrm{TaC}$ and 2-STC switches. For this purpose we shall compare integrated optics implementations of the three models.

\section{A. Power Loss of the SaD-Based Node}

The $\mathrm{SaD}$ switch is proposed to be made up of $P$ configurable splitters [4]. We propose a configurable splitter by cascading $2 \times 2$ MMI's with symmetric splitting ratios. In a single $2 \times 2$ MMI with symmetric splitting ratios [13] the output power from one input can be tuned to the other by biasing the refractive index in specific sections of the MMI. According to simulations [13], it has output ratios of 50:50 (i.e., a single split, initial state) and $0.1 \mathrm{~dB}$ excess losses with no biasing. But when it is biased to obtain 0:100 or 100:0 split ratios, it actually obtains $1: 71$ and $71: 1$ split ratios, mainly because of the attenuation losses caused by biasing. Therefore, for a switched signal simulation losses are around $1.55 \mathrm{~dB}$. As an example, we present a possible implementation of a $1 \times 4$ configurable splitter in Fig. 8. In general, it would be necessary to align $(P-1) 2 \times 2$ MMI's with symmetric splitting ratio in cascade, arranged in $\log _{2} P$ phases ( $P=$ number of ports). Depending on the operation needed, the input power would incur in different losses (see Table I).

Loss grows with the number of ports because the number of phases also increase. For instance, for a $1 \times 8$ configurable splitter, 3 phases will be needed and losses will be in the order of $4.65 \mathrm{~dB}$ for a single switch operation in continue mode. For a
$1 \times 16$ (or $1 \times 12$ ) device ( 4 phases), losses will be in the order of $6.2 \mathrm{~dB}$ for a single switch; and so on.

Besides the power losses caused by configurable splitters, a signal has to traverse an optical switch matrix (see Fig. 1(a) for reference), which contributes to overall losses. Therefore, power losses (in $\mathrm{dB}$ ) of an input signal in $\mathrm{SaD}$ switch are given by

$$
\begin{aligned}
\mathrm{PL}_{\mathrm{SaD} \text { switch }}= & \left(N_{\mathrm{MMI}-\text { bias }} \cdot \mathrm{PL}_{\mathrm{MMI}-\text { bias }}\right) \\
& +\left(\log _{2} P-N_{\mathrm{MMI}-\text { bias }}\right) \\
& \cdot\left(\mathrm{PL}_{\mathrm{MMI}-\text { no-bias }}\right) \\
& +10 \log _{10} m+N\left(\mathrm{PL}_{\mathrm{Sw}}\right)
\end{aligned}
$$

where $P$ is the number of ports, $N_{\mathrm{MMI} \text {-bias }}$ is the number of tunable $2 \times 2$ MMI splitters, $\mathrm{PL}_{\mathrm{MMI}-\text { bias }}$ is the power loss of a tunable $2 \times 2 \mathrm{MMI}$ splitter when changing power ratios to $0: 100$ or 100:0, $\mathrm{PL}$ MMI-no-bias is the power loss of a tunable $2 \times 2$ MMI splitter when not biased, $m$ is the number of split outputs, $N$ is the amount of optical switches the signal has to traverse at the switch matrix and $\mathrm{PL}_{\mathrm{sw}}$ is the insertion loss of a $2 \times 1$ optical switch.

Finally, the overall loss of an input signal in a SaD-based node [see Fig. 1(b)] is

$\mathrm{PL}_{\mathrm{SaD} \text {-based node }}=\mathrm{PL}_{\mathrm{dm}}+\mathrm{PL}_{\mathrm{SaD} \text { switch }}+\mathrm{PL}_{\mathrm{mx}}(\mathrm{dB})$

where $\mathrm{PL}_{\mathrm{dm}}$ and $\mathrm{PL}_{\mathrm{mx}}$ are the power losses caused by demultiplexing and multiplexing, respectively. The expression (3) is given only as a reference, but since the power losses for demultiplexing and multiplexing are the same for $\mathrm{SaD}, \mathrm{TaC}$ and 2-STC architectures, we do not take them into account, because they do not make any difference in the comparison.

\section{B. Power Loss of the TaC Node}

In the case of TaC nodes (see Fig. 2), losses are caused mainly by the optical switching elements of the WRS and TCM modules. Notice that when tapping, the signal has to traverse $\log _{2} P$ optical switches. Therefore, losses are

$$
\begin{aligned}
\mathrm{PL}_{\mathrm{TaC} \text { node }}=\left(N_{\mathrm{WRS}}\right. & \left.+B \cdot \log _{2} P+1\right) \cdot\left(\mathrm{PL}_{\mathrm{sw}}\right) \\
& +B \cdot \mathrm{PL}_{\mathrm{tap}}+\mathrm{PL}_{\mathrm{dm}}+\mathrm{PL}_{m}(\mathrm{~dB})
\end{aligned}
$$

where $P$ is the number of ports, $\mathrm{PL}_{\mathrm{dm}}, \mathrm{PL}_{m x}$ and $\mathrm{PL}_{\mathrm{sw}}$ have the same meaning as in expressions (2) and (3), $N_{\text {WRS }}$ is the number of switches the signal has to traverse in the WRS module, $B$ is a boolean variable (equal to 1 when the tap action is performed, otherwise is 0 ) and $\mathrm{PL}_{\text {tap }}$ is the power loss of the fraction tapped to the local node, given in $\mathrm{dB}$.

\section{Power Losses of the 2-STC Node}

To analyze the 2-STC node we need to model its main component, the Ta2S switch. A description of the Ta2S switch behavior has been done using Matlab. Block diagram of the proposed MZI switch is shown in Fig. 5(a). The device behavior is determined by the expressions (5)-(8), shown at the bottom of the next page, where $P_{\mathrm{IN}}, P_{\mathrm{BAR}}$ and $P_{\mathrm{CROSS}}$ are optical powers at the input, bar and cross output respectively, Tap is the optical portion of the power needed for the tap operation, $\gamma_{\text {tap }}, \gamma_{1}$ and 
$\gamma_{2}$, are the excess losses of each MMI coupler, $\kappa_{1}$ and $\kappa_{2}$ are the coupling ratios, $L$ is the waveguide length, $\alpha$ is the attenuation of the waveguide in Neppers/m, $\Delta \alpha$ is the attenuation increment due to the variation of the refractive index in Neppers $/ \mathrm{m}, \Delta \beta$ is the change of the propagation constant due to refractive index change, $\Delta n$, and $\lambda_{0}$ is the vacuum wavelength.

When the refractive index is modified, an increment of the attenuation loss is expected. The increment of attenuation simulated is accorded to the equation given in [13] for free-carrier-absorption when current injection is used to change the refractive index.

The necessary $\Delta n$ for optical switching is obtained by solving (5) when bar output is 1 , considering that there are no losses when the MMI is switched

$$
\Delta n=\frac{\lambda_{0}}{2 \cdot L} .
$$

The obtained refraction index variation necessary for switching a $100 \mu \mathrm{m}$ length waveguide MZI is $\Delta n=$ $-7.75 \cdot 10^{-3}$. Simulations have been made with a $6 \%$ tap, 0.1 $\mathrm{dB}$ excess loss for each MMI, coupling ratios $\kappa_{1}=\kappa_{2}=0.5$, $100 \mu \mathrm{m}$ waveguide length $(L)$, and $1.55 \mu \mathrm{m}$ vacuum wavelength.

Output powers of the Ta2S switch as a function of refractive index variations at ideal (lossless) and real case are shown in Fig. 9(a). Excess losses about $0.7 \mathrm{~dB}$ and $0.55 \mathrm{~dB}$ are obtained for bar and cross outputs, respectively, when they are active. Insertion losses are about $3.7 \mathrm{~dB}$ when switch is in (50:50) configuration. A fabrication tolerance error of $0.1 \%$ in both MMI coupling ratios, $\kappa_{1}$ and $\kappa_{2}$, has also been considered in simulations. A detail of the bar output in active state is shown in Fig. 9(b).

Once the insertion losses for the different states of the Ta2S switch have been modeled, we can give the expression for loss in the complete 2-STCM module (see Fig. 7) as

$$
\begin{aligned}
\mathrm{PL}_{2-\mathrm{STCM}}= & \mathrm{PL}_{\mathrm{Ta} 2 \mathrm{~S}}+10 \log _{10} m \\
& +\left(\log _{2} P+1\right) \cdot \mathrm{PL}_{s w 2 \times 1 / 2 \times 2} \\
\text { and } & \mathrm{PL}_{\text {cross }} \\
\mathrm{PL}_{\text {Ta2S }}= & \mathrm{PL}_{\text {tap }}+\left\{\begin{array}{l}
\text { bar } \\
\mathrm{PL}_{2-\text { split }}
\end{array}\right.
\end{aligned}
$$

where $\mathrm{PL}_{\mathrm{Ta} 2 \mathrm{~S}}$ is the power loss when the signal traverses a Ta2S, $P$ is the number of output ports and $m$ is the split factor

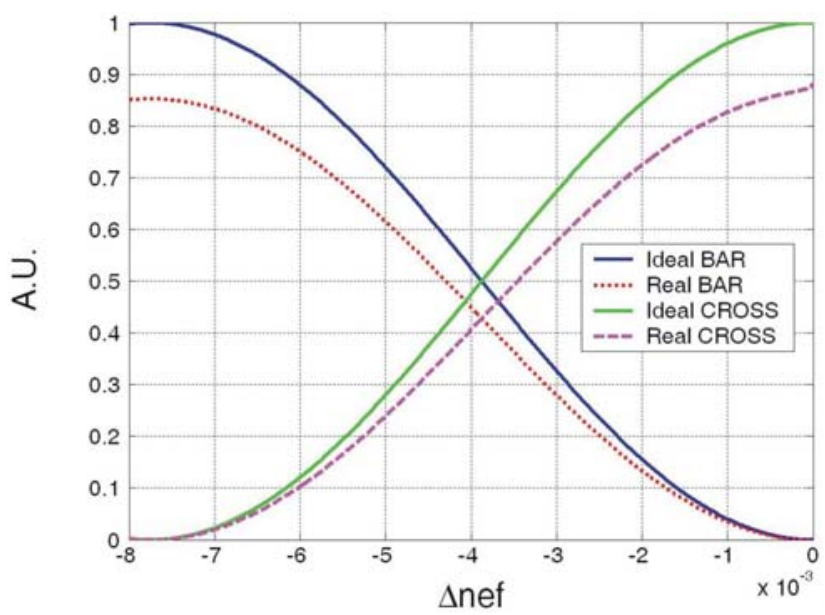

(a)

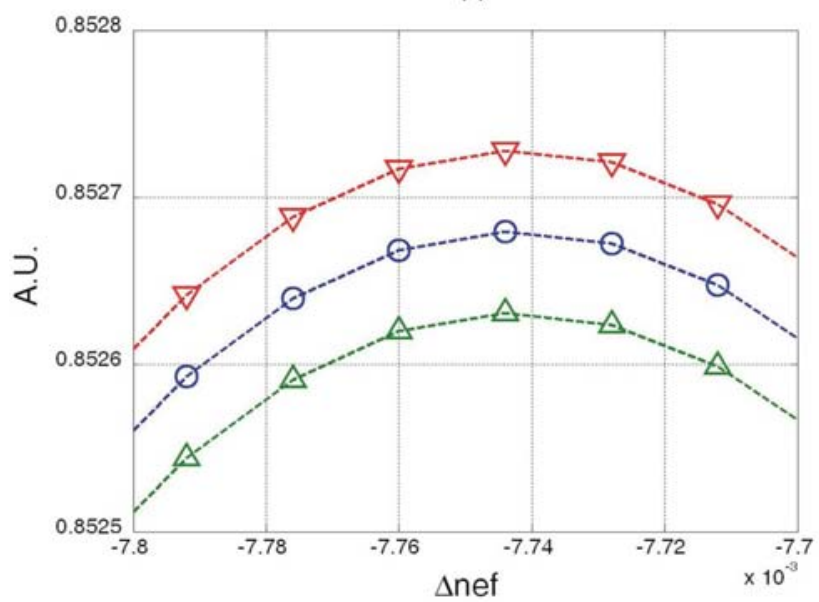

(b)

Fig. 9. Simulation results for a MZI optical switch with fixed tap. (a) Cross and bar outputs of the node versus refractive index changes. (b) Difference in bar output power at $\Delta \mathbf{n}=-7.75 \cdot 10^{-3}$ when $0.1 \%$ error in $\kappa$ is considered.

(1 or 2 ), $\mathrm{PL}_{\mathrm{sw} 2 \times 1 / 2 \times 2}$ is the power loss incurred by an optical $1 \times 2$ or $2 \times 2$ switching element. $\mathrm{PL}_{\text {cross }}$ and $\mathrm{PL}_{\text {bar }}$ are losses in cross or bar states and $\mathrm{PL}_{2-\text { split }}$ is the excess loss when performing 2-splitting.

\section{Comparison of Number of Components and Power Loss}

In Table II and in Fig. 10(a) we can see that our proposal scales conveniently with respect to $\mathrm{SaD}$ and $\mathrm{TaC}$ modules. The number of components (optical switches, Ta2S switches, tunable $2 \times 2$ MMI splitters and tap devices) in $2-\mathrm{STCM}$ is much

$$
\begin{aligned}
\left|\frac{P_{\mathrm{BAR}}}{P_{\mathrm{IN}}}\right| & =\Gamma \cdot\left[\begin{array}{c}
\left(1-\kappa_{1}\right) \cdot\left(1-\kappa_{2}\right) \cdot e^{-2 \cdot \Delta \alpha \cdot L}+\kappa_{1} \cdot \kappa_{2} \\
-2 \cdot \sqrt{\left(1-\kappa_{1}\right) \cdot\left(1-\kappa_{2}\right) \cdot \kappa_{1} \cdot \kappa_{2}} \cdot e^{-\Delta \alpha \cdot L} \cos (\Delta \beta \cdot L)
\end{array}\right] \\
\left|\frac{P_{\mathrm{CROSS}}}{P_{\mathrm{IN}}}\right| & =\Gamma \cdot\left[\begin{array}{c}
\left(1-\kappa_{1}\right) \cdot\left(1-\kappa_{2}\right) \cdot e^{-2 \cdot \Delta \alpha \cdot L}+\kappa_{1} \cdot \kappa_{2} \\
+2 \cdot \sqrt{\left(1-\kappa_{1}\right) \cdot\left(1-\kappa_{2}\right) \cdot \kappa_{1} \cdot \kappa_{2}} \cdot e^{-\Delta \alpha \cdot L} \cos (\Delta \beta \cdot L)
\end{array}\right] \\
\Gamma & =(1-\mathrm{Tap}) \cdot\left(1-\gamma_{\mathrm{Tap}}\right) \cdot\left(1-\gamma_{1}\right) \cdot\left(1-\gamma_{2}\right) \cdot e^{-2 \cdot \alpha \cdot L} \\
\Delta \beta & =\frac{2 \cdot \pi}{\lambda_{0}} \cdot \Delta n
\end{aligned}
$$


TABLE II

COMPONENTS USED IN INTERNAL SWITCH MODULES

\begin{tabular}{lcccc}
\hline & $\mathrm{SaD}$ & $\mathrm{TaC}$ & $\mathrm{ns}-\mathrm{TaC}$ & 2-STCM \\
\hline Switches & $P^{2}$ & $P^{2}+2 P-1$ & $P^{2}$ & $P \log _{2} P+\frac{P}{2}$ \\
$(2 \times 2 / 2 \times 1)$ & & & & $*$ \\
& & & - & $P$ \\
MZI switches & - & - & - & - \\
Tunable $2 \times 2$ & $P(P-1)$ & - & & $P$ \\
MMI splitters & & 1 & $P$ & \\
Tap devices & - & & & \\
\hline
\end{tabular}

${ }^{*}$ This value is valid if $P$ is a power of 2 , otherwise it equals

$\left(\log _{2} P^{\prime}\right)\left(2 P-P^{\prime}\right)+\frac{P}{2}$, where $P^{\prime}=2^{\left\lceil\log _{2} P\right\rceil}$

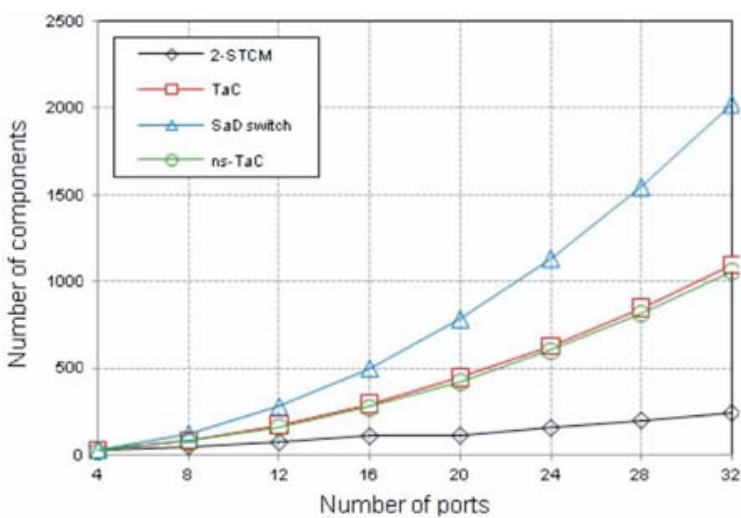

(a)

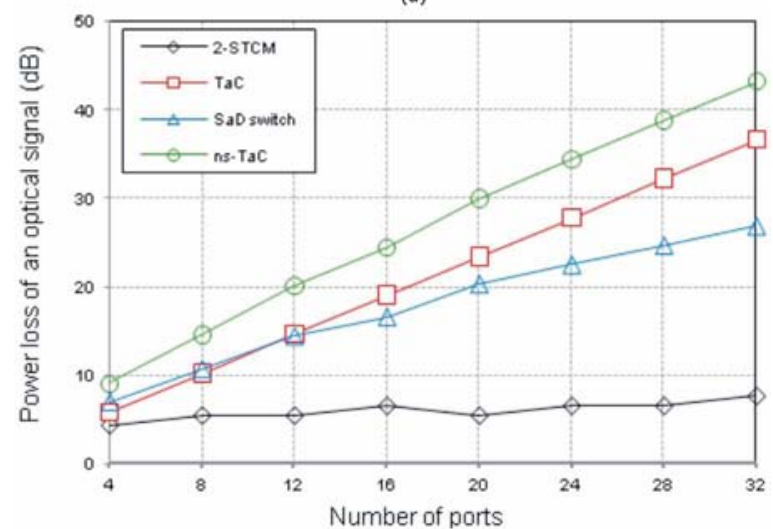

(b)

Fig. 10. Comparison of architectures. (a) Total number of components in 2-STCM, TaC and SaD modules. (b) Power loss (in $\mathrm{dB}$ ) of a single optical signal for 2-STCM, TaC and $\mathrm{SaD}$ switch modules when a tap/drop-and-continue action is performed (average case)

lower than the other proposals, which contributes to improve the power efficiency.

We shall make an overall assessment of power loss in a network by means of a concrete example. Let us assume that $51 \mathrm{~dB}$ (see [6]) is the sample power budget, i.e., although ideally amplification can compensate loss, attenuation must not exceed the target power budget for the receiver to work properly. We have calculated the power loss incurred by a single optical signal when continue, tap-and-continue, 2-split, and tap-and-2-split actions are taken in these three modules for best, average and worst cases. For the purpose of comparison, no amplification is considered in any of the nodes.
In order to estimate the power loss, we have considered that a single $1 \times 2$ or $2 \times 1$ optical switch has a power loss of $1.1 \mathrm{~dB}$. $\mathrm{TaC}, \mathrm{SaD}$ and 2-STCM have been compared for continue and tap-and-continue operations (in the case of $\mathrm{SaD}$, it is a dropand-continue action), and results are presented in Fig. 10(b) for the average case. Fig. 10(b) also depicts the power loss incurred by a non-shared-tap-TaC (ns-TaC) node for the same setting. This architecture is introduced in the comparison to have also the cost of tap-sharing stripped off regular $\mathrm{TaC}$ [7]. Note that this cost is not negligible as the need to re-switch the continue signal to an output port requires additional switching elements (see Fig. 2). This could be avoided by tapping each input as done in 2-STCM. Then, a ns-TaC would have $P$ Tap devices but just $P^{2} /, 2 \times 2$ switches.

Fig. 10 shows that 2-STCM has better scalability in terms of number of components and power efficiency for a $\mathrm{TaC}$ operation. Although $\mathrm{TaC}$ may use fewer components than $\mathrm{SaD}$, it performs the worst in power efficiency. We also observed the same trend for the continue action.

If optical amplification inside the node was considered, the 2-STC node is the most convenient option, because it would use much fewer amplifiers $(2 P)$ than $\mathrm{SaD}\left(P^{2}\right)$ but more than $\mathrm{TaC}(P)$. Since best, average, and worst cases are the same for switching in 2-STCM, losses may be known in advance and precise required amplification levels can be set up beforehand. In the $\mathrm{SaD}$ switch, power loss grows with the number of ports as $10 \cdot \log (m)$, whereas losses in 2-STCM grows at a lower rate.

\section{EVALUATION OF APPLICABILITY}

In the previous section, it has been shown that 2-STC nodes present better power efficiency than the other architectures in tap-and-continue mode when implemented with integrated optics as described. However it should be clearly stated that globally $\mathrm{TaC}$ is more power-efficient than 2-STC and $\mathrm{SaD}$ when the nodes are set in split mode. The insertion loss due to splitting is far from that of tapping. The price that $\mathrm{TaC}$ pays is the lack of optimality in the delivery graph i.e., more link wavelengths are taken to reach all target nodes.

In this sense, one major issue to be analyzed is whether the 2-split constraint of 2-STC is enough in practice to satisfy multicast requests in a wavelength-efficient way. In principle, since most core network topologies have an average degree between 3 and 4, binary-split should perform quite well.

Therefore, we evaluate how well 2-STC nodes perform on a reference core network. We took several metrics and compared them under different multicast requests in a network topology made up of 28 nodes: the COST266 Optical Transport reference network (http://www.ibcn.intec.ugent.be/INTERNAL/NRS/index.html), shown in Fig. 11. Since some nodes of this network have more than 4 output links, we consider that all nodes have 8 ports.

\section{A. LightTree-Making Algorithms}

In order to compare the three architectures under study, we need to implement tree-construction algorithms for each one. The three target problems are known to be NP-Complete as shown in the references that follow. These problems are: a) for 


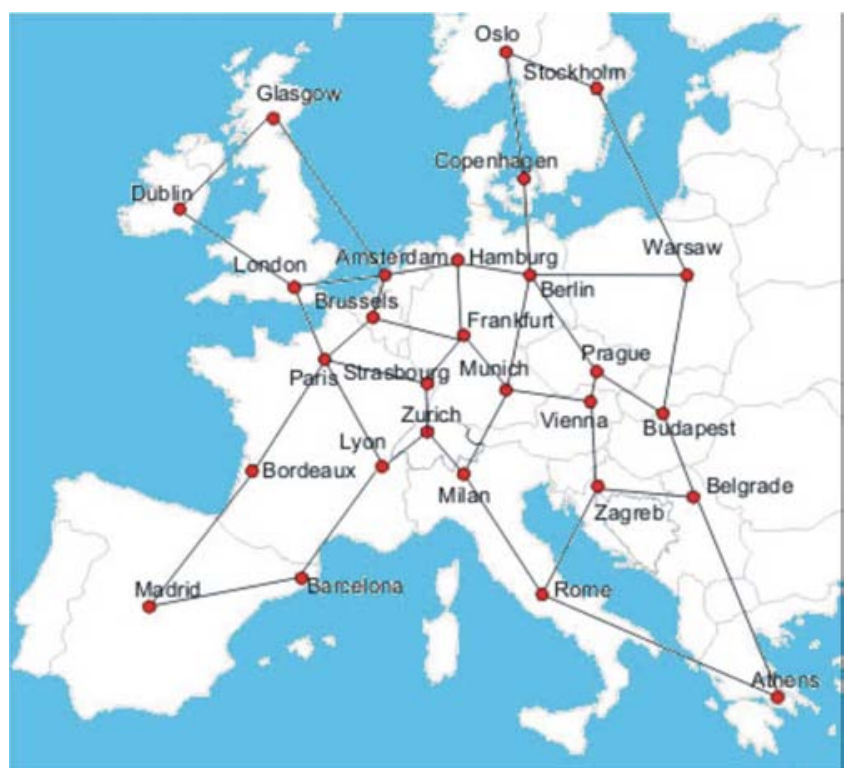

Fig. 11. COST266 Optical Transport Reference Network.

$\mathrm{SaD}$ : Steiner tree with no degree constraint, i.e., the generic Steiner Tree Problem [16]; b) for 2-STC: degree-constrained Steiner tree [17], in particular the degree constraint is 4 (one input plus three outputs (tap and binary split)); and finally c) for TaC: a variant of the "open traveling salesman problem" [7] where only a subset of nodes in the network needs to be visited and there is no need to return to the origin.

Since this paper is not actually focused on tree optimization and it is not practical to compute the optimal spanning tree solution even for medium-size networks, we shall use existing heuristics where available. Intensive simulations show that very simple heuristics can provide cost-effective solutions not far from optimal [17]. Thus, for a) ( $\mathrm{SaD}$ ) we shall simply use the Shortest Path Tree given by Dijkstra as a simple and realistic heuristic for tree construction. This is the type of tree that IP multicast routing protocols such as MOSPF or PIM-DM produce.

In [7] the authors describe their target problem as the Multiple-Destination Minimum-Cost Trail (MDMCT) problem for their $\mathrm{TaC}$ architecture. Therefore, for c) ( $\mathrm{TaC})$ we shall use the algorithm proposed in [7], which has been designed with the guarantee that no link is traversed more than once on a given direction. This is claimed by the authors to perform better than other approaches such as next-nearest node first.

Finally, for b) (2-STC) we propose an heuristic algorithm inspired in the informal descriptions of [17] that removes the constraint of "no return to a previously visited node" of [17]. It should be noted that solving special cases with 2-STC, and also with $\mathrm{TaC}$, requires back-tracking. In this sense, the worst case example for both $\mathrm{TaC}$ and 2-STC is the star topology. With respect to $\mathrm{SaD}$, broadcasting in an $N$-node star net requires $N$ links, $N-2$ additional hops with $\mathrm{TaC}$ and $(N-3) / 2$ additional hops using 2-STC (value for $N=2^{k}-1$, with $k$ integer). In practice, core networks are usually 2 -connected for resilience reasons, and backtracking over the same link could be prevented.
The main challenge for an algorithm able to work with 2-STC nodes is to convert a light-tree into a binary light-tree, where every node can not have more than two out links. In the following description of the algorithm (BinaryTree function), the Steiner tree for the multicast session is given as an input. Basically, this algorithm analyses nodes with more than two children and finds possible alternative paths in order to accomplish the 2 -split constraint for the parent node. The path is chosen so as to try to get the shortest distance and avoiding other nodes to have more than two children. If more than one solution is obtained it is selected randomly. In the case that it is not possible to obtain any other path, a special round-trip path is established. To achieve this, the links with the shortest distance are chosen and a path of the type childrenA-parent-childenB is established.

Once the solution is found, the light-tree has to be recalculated (recalculateTree function in the algorithm), i.e., distances, children and links must be reconsidered with the new path-branch found.

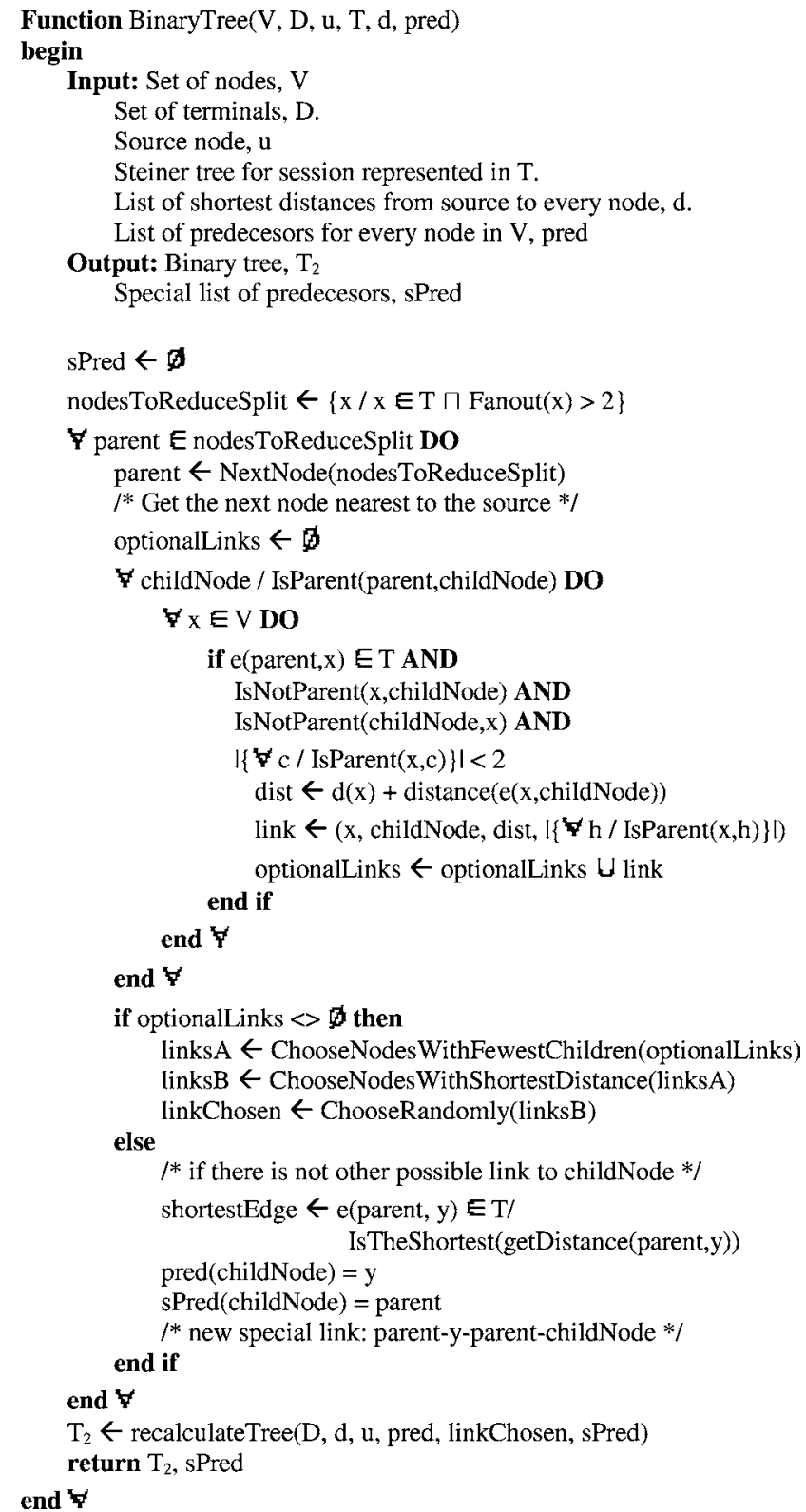




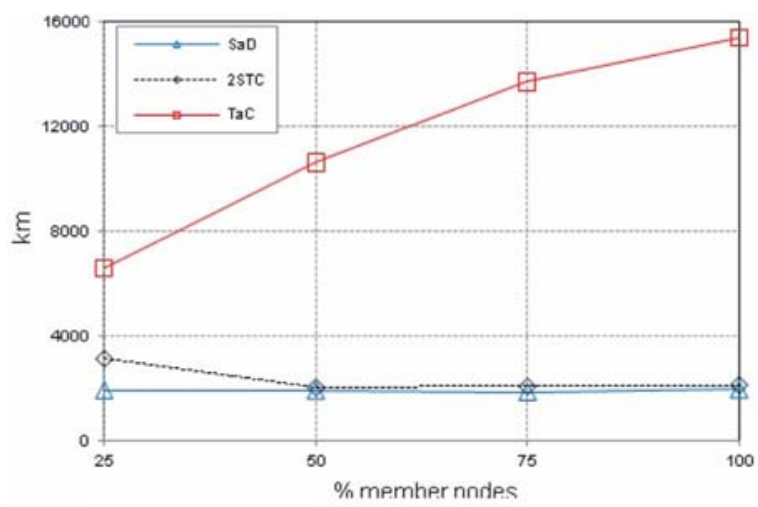

(a)

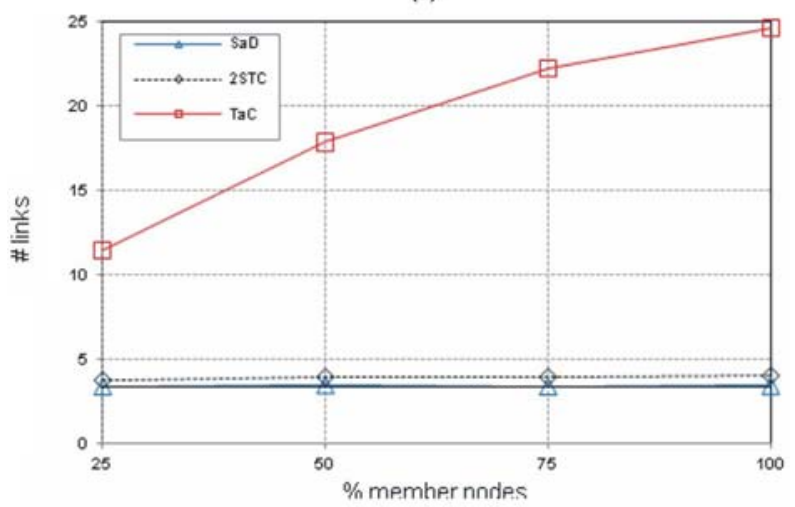

(b)

Fig. 12. Average lightpath length. (a) Average root-to-destinations distance. (b) Average root-to-destinations number of links.

\section{B. Simulation Methodology}

We ran a series of simulation experiments in Matlab 7.0 over the reference topology in Fig. 11, [18], including physical distance between nodes. Each single experiment consisted of a multicast request made up of a random root and a random subset of multicast members. The simulator built the trees for $\mathrm{SaD}$, $\mathrm{TaC}$ and 2-STC for each random request, using the algorithms described previously. The experiments were grouped into series of different densities of receivers in the network. Thus, four separate experiment series corresponding to random multicast requests spanning $25 \%, 50 \%, 75 \%$ and $100 \%$ receivers in the network were executed. For each series, we measured: (a) the average root-to-destinations distance, (b) the average root-to-destinations number of links, and (c) the average power per link, not having into account link attenuation. We wish to focus solely on the comparison of loss due by the different node architectures. Again, no amplification is considered for any of the architectures compared. The simulation was stopped when the target average $X$ and confidence interval of $95 \%, X \pm \Delta X$, held: $\Delta X /|X| \leq 5 \%$. This interval is not represented in the graphs for the sake of clarity.

\section{Source-Destination Distance and Link Consumption}

Fig. 12 shows the average source-to-destination distance and the average source-to-destination link consumption resulting from the simulation for TaC, 2-STC and SaD. In a TaC-node network, the resulting light-tree should actually be a single

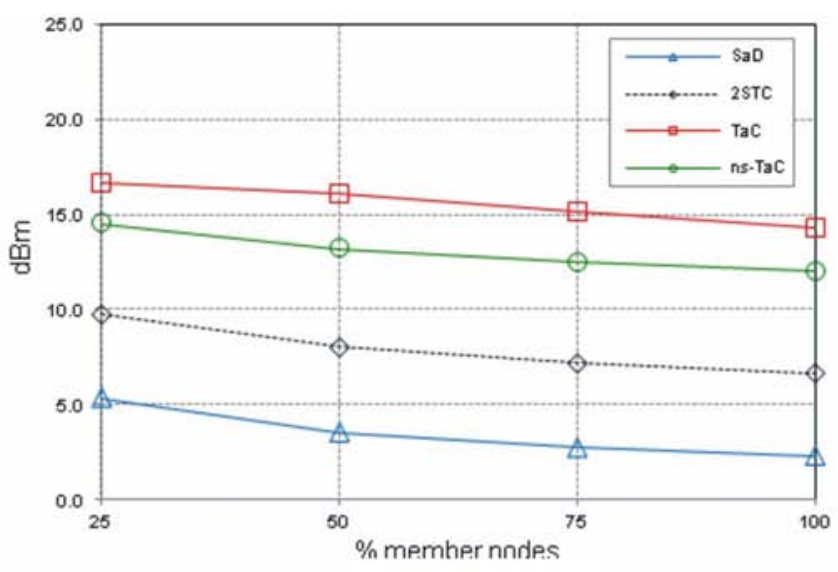

(a)

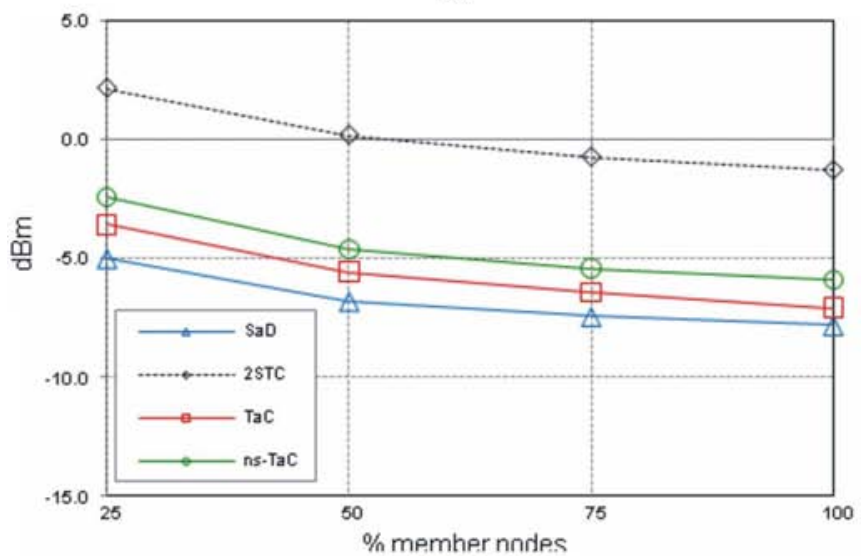

(b)

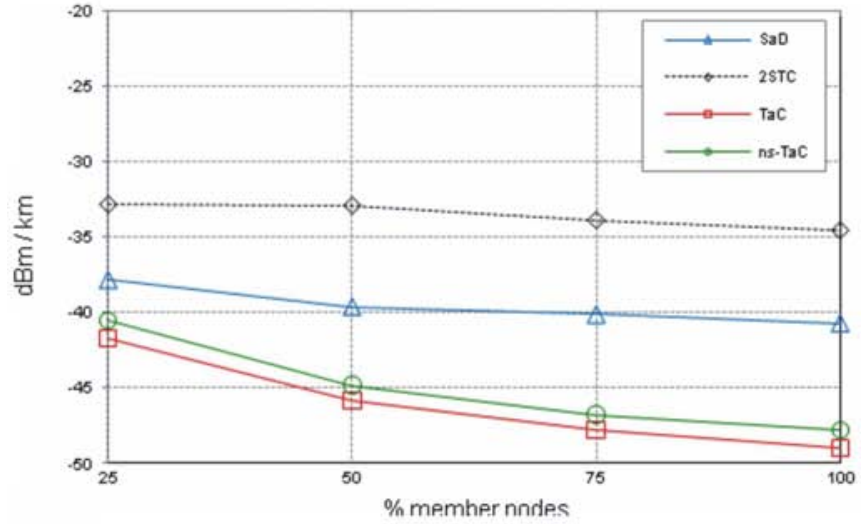

(c)

Fig. 13. Average input power. (a) Average incoming power at nodes without intern node attenuation. (b) Average incoming power at nodes with intern node attenuation. (c) Ratio of average incoming power per kilometer $(\mathrm{dBm} / \mathrm{km})$.

lightpath that traverses the minimum possible number of nodes to pass through all destination nodes, where a TaC operation is performed. Therefore, the distance and absolute link use of $\mathrm{TaC}$ is well above 2-STC and $\mathrm{SaD}$. The graph also shows that the more dense the tree is, the longer the average path in number of hops and in distance. Thus, $\mathrm{TaC}$ seems not to be a good option for broadcasting in a big meshed network in terms of delay and resource consumption. Some improvement to this result could be expected from better heuristics to the open traveling salesman problem, although always performing worse than a tree. The picture also reveals that the degree constraint to 
multipoint of 2-STC makes almost no difference to SaD in this topology, which was expected to perform better than 2-STC, even though some nodes have 5 links. In other words, the resource consumption is almost the same with binary light-trees. The result confirms previous works [17] on experiments with thousands of random topologies, where the authors conclude that the importance of unconstrained multipoint capability (both in terms of \% of node support and maximum fan-out) is usually overestimated.

\section{Power}

A key parameter in the evaluation of the architectures under study is the average power that arrives at each node. As regards tapping versus splitting, TaC must have much better performance than 2-STC and SaD. This effect is illustrated in Fig. 13(a), where all attenuation due to switching inside the node has been omitted. Even though the path to run by $\mathrm{TaC}$ and the number of hops is larger than in the other approaches, the hard loss due to splitting is predominant.

However, ns-TaC and TaC need a $P \times P$ switching fabric whose attenuation is very relevant (above $4 \mathrm{~dB}$ in average for a $4 \times 4$ switch), given the extra nodes to be traversed w.r.t. $\mathrm{SaD}$ and 2-STC (see previous subsection). Furthermore, TaC has an additional modul77e to re-switch the continue signal. In contrast, $\mathrm{SaD}$ and 2-STC have a more power-efficient mesh of optical switches after splitting. This fact leaves $\mathrm{TaC}$ in a situation quite comparable to the splitting-based approaches.

The results from simulation in Fig. 13 show that it is exactly the case in our scenario. Given its required number of hops and attenuation due to switching, TaC gives the lowest average power per link. If tapping is not shared, the ns-TaC case, then the average power is over that of $\mathrm{SaD}$, which implicitly means a lesser need for amplification. 2-STC performs slightly better in this scenario. This improvement is amplified by the fact that 2-STC has a similar link consumption as $\mathrm{SaD}$ in practice. Fig. 13(b) shows this effect.

It should be remarked again the importance of a good heuristic for tree construction in $\mathrm{TaC}$ and of the topology. Smaller networks and topologies more favorable to TaC, like ring or multiple-ring-based topologies, are not expected to show such a difference. Regarding SaD versus 2-STC, it is clear that for the reference integrated optics implementation that we have used, 2-STC outperforms $\mathrm{SaD}$ in power-efficiency and the extra link consumption of 2-STC seems not to be relevant.

\section{CONCLUSION AND FUTURE WORK}

In this paper, we have presented a MC-OXC node called 2-STC, that performs $\mathrm{TaC}$ and $\mathrm{Ta} 2 \mathrm{~S}$ operations to support multicast in WDM networks and is efficient and scalable in terms of number of components. Moreover, the design shows relevant improvement in power efficiency over $\mathrm{SaD}$ and $\mathrm{TaC}$. When used in Tap and Continue mode it features less attenuation than the tap-shared (regular) TaC node in isolation, but also less than $\mathrm{ns}-\mathrm{TaC}$ in a networked simulation. When used in tap and binary-split mode, the 2-STC combines the properties of both $\mathrm{TaC}$ and $\mathrm{SaD}$ to reduce the need for optical amplification. The 2-STC is based on 2-STCM modules whose main component is a novel Tap-and-2-Split Switch which has been designed using MMI taps and MZI switches. The architecture features an original binary-split plus parallel switch \& merge approach based on a novel interconnection network which is the key for its efficiency.

The simulations illustrate that the binary-splitting constraint of 2-STC has a reduced impact on optimality. Indeed, the network can serve any multicast request in typical network topologies at a very small additional link cost, thus achieving an interesting trade-off between power efficiency and network resource consumption.

The paper focuses on the analysis of power loss in the three architectures under study using a similar integrated optics implementation. Other type of technologies should also be studied in the future, as well as improved algorithms for TaC and 2-STC light-tree construction.

\section{REFERENCES}

[1] Y. Zhou and G.-S. Poo, "Optical multicast over wavelength-routed WDM networks: A survey," Optical Switching Netw., vol. 1, 2005.

[2] L. H. Sahasrabuddhe and B. Mukherjee, "Light-trees: Optical multicasting for improved performance in wavelength-routed networks," IEEE Commun. Mag., vol. 37, no. 2, pp. 67-73, Feb. 1999.

[3] G. N. Rouskas, "Optical layer multicast: Rationale, building blocks, and challenges," IEEE Network, vol. 17, pp. 60-65, 2003.

[4] W. S. Hu and Q. J. Zeng, "Multicasting optical cross connects employing splitter-and-delivery switch," IEEE Photon. Technol. Lett., vol. 10, no. 7, pp. 970-972, Jul. 1998.

[5] M. Ali and J. Deogun, "Power-efficient design of multicast wavelengthrouted networks," IEEE J. Sel. Areas Commun., vol. 18, no. 10, pp. $1852-1862,2000$.

[6] G. N. Rouskas, "Light-tree routing under optical layer power budget constraints," in Proc. 17th IEEE Comp. Commun. Wksp., 2002, pp. $14-16$.

[7] M. Ali and J. Deogun, "Cost-efficient implementation of multicasting in wavelength-routed networks," J. Lightw. Tech., vol. 18, no. 12, pp. 1628-1638, Dec. 2000.

[8] H. Zang, J. P. Jue, and B. Mukherjee, "A review of routing and wavelength assignment approaches for wavelength-routed optical WDM networks," Opt. Net., vol. 1, no. 1, pp. 47-60, Jan. 2000.

[9] Y. Xin and G. N. Rouskas, "Multicast routing under optical layer constraints," in Proc. IEEE Infocom, 2004, vol. 4, pp. 2731-2742.

[10] K.-D. Wu, J.-C. Wu, and C.-S. Yang, "Multicast routing with power consideration in sparse splitting WDM networks," in Proc. IEEE ICC, 2001, pp. 513-517.

[11] J. Y. Feng, T. S. Lay, and T. Y. Chang, "Waveguide couplers with new power splitting ratios made possible by cascading of short multimode interference sections," Opt. Exp., vol. 15, no. 4, pp. 1588-1593, 2007.

[12] C. Vázquez, F. J. Mustieles, and J. F. Hernández Gil, "Three-dimensional method for simulation of multimode interference couplers," $J$. Lightw. Technol., vol. 13, no. 11, pp. 2296-2299, Nov. 1995.

[13] J. Leuthold and C. H. Joyner, "Multimode interference couplers with tunable power splitting ratios," J. Lightw. Technol., vol. 19, no. 5, pp. 700-706, May 2001.

[14] C. Vázquez, S. Vargas, S. Pena, and J. M. , "Sagnac loop in ring resonators for tuneable optical filters," J. Lightw. Technol., vol. 23, no. 8, pp. 2555-2567, Aug. 2005.

[15] G. M. Fernández, D. Larrabeiti, C. Vázquez, and P. C. Lallana, "Power-cost-effective node architecture for light-tree routing in WDM networks," IEEE Globecom2008 [Online]. Available: http://www.it.uc3m.es/dlarra/out/globecom2008/p.pdf

[16] F. K. Hwang, D. S. Richards, and P. Winter, The Steiner Tree Problem. New York: Elsevier, 1992, vol. 53.

[17] F. Bauer and A. Varma, "Degree-constrained multicasting in point-topoint networks," in Proc. IEEE INFOCOM'95, Boston, MA, 1995, pp. 369-376.

[18] S. de Maesschalck et al., "Pan-european optical transport networks: An availability-based comparison," Photonic Netw. Commun., vol. 5, no. 3, pp. 203-225, 2003. 
Gonzalo M. Fernandez-del-Carpio currently preparing his Ph.D. dissertation on optical multipoint networking architectures at University Carlos III of Madrid, Madrid, Spain, with the support of a Fundacion Carolina grant and the BONE network of excellence.

He is currently a Professor at University Catolica de Peru at Arequipa, Peru.

Carmen Vázquez received the M.Sc. degree in physics (electronics) in 1991 from the Complutense University of Madrid, Madrid, Spain, and the Ph.D. degree in physical sciences from the Universidad Politécnica of Madrid, Madrid, Spain, in 1995, carrying out her doctoral studies in the department of photonics of the E.T.S.I. Telecomunicación (Higher Technical School of Telecommunications Engineering).

In 1991 she worked as an intern in the Danish company TELECOM (Copenhagen) and from 1992 to 1995 worked in the Optoelectronics division of "Telefónica Research and Development" in Madrid. During this time Dr. Vazquéz participated in design projects, and analysis of integrated optical devices. She joined Carlos III University in 1995, and is an Associate Professor and head of the Displays and Photonic Applications Group. She was also Head of the Department of Electronic Technology for 3 years. Her research interests focus on optical communications and instrumentation, including optical signal processing, ring resonators, plastic fiber optics, broadband access networks, filters, switches, fiber optic sensors and WDM networks. She has published more than 35 studies in prestigious international journals and given many conferences, with more than 70 citations. She has several patents of optical sensors. Dr. Vázquez has participated in different European projects and networks since 1992 in the ESPRIT, RACE and IST framework programmes such as PLANET, OMAN, HEMIND, SAMPA, EPhoton/One+, etc., as well as in the FACTOTEM macro-groups of the Madrid Autonomous Community. She has headed several projects within the National Plan and autonomous communities related to fiber optic sensor networks and broadband photonic devices: FOTOCOMIN, SAFE-OF, etc.
Pedro Contreras Lallana received the M.Sc. degree in telecommunication engineering from the Technical University of Madrid (UPM), Madrid, Spain, in March 2003.

He is working as a Teaching Assistant at the Electronics Technology Department of Carlos III University of Madrid. As a member of the Displays and Photonic Applications Group, he research is involved in integrated optics, advanced devices based on fibers and liquid crystals for WDM networks.

David Larrabeiti received the M.Sc. and Ph.D. degrees in telecommunication engineering from Technical University of Madrid (UPM), Madrid, Spain, in 1991 and 1996, respectively.

He is a Professor at Universidad Carlos III de Madrid (UC3M, Spain), where he has been teaching and doing research on high-speed network and switching architectures for ten years. He has participated in many international research projects on next generation networks. He is currently the responsible for BONE EU network of excellence on optical networks at UC3M. His research interests include the design of future Internet infrastructure, ultra-broadband multimedia transport, and traffic engineering of IP-G/MPLS networks. 